Palavras chave: Mineração de dados Ecologia da paisagem GeoDMA

Histórico:

Recebido 26/10/2014 Aceito 18/12/2015

Keywords: Data Mining Landscape ecology GeoDMA

Correspondência: nssaito@dsr.inpe.br
Nathália Suemi Saito' , Fernanda Viana Paiva Arguello', Maurício Alves Moreira', Alexandre Rosa dos Santos ${ }^{2}$, Fernando Coelho Eugenio ${ }^{2}$, Alvaro Costa Figueiredo ${ }^{2}$

\section{USO DA GEOTECNOLOGIA PARA ANÁLISE TEMPORAL DA COBERTURA FLORESTAL}

RESUMO: As métricas de ecologia da paisagem associadas à mineração de dados podem ser utilizadas para aumentar o potencial de análise e aplicações de dados de sensoriamento remoto, tornando-se uma importante ferramenta para a tomada de decisão. Dessa forma, objetivou-se classificar e quantificar diferentes tipos de vegetação por meio de técnicas de mineração de dados e métricas de ecologia da paisagem em uma análise multitemporal (200 I e 20 I I), em São Luís do Paraitinga, São Paulo, Brasil. A análise de imagens orientada a objetos e o algoritmo de mineração de dados $C 4.5$ foram utilizados para realizar a classificação automática, cuja precisão foi avaliada com o índice kappa e com as medidas de discordância de alocação e de quantidade, recentemente propostas na literatura. Foram classificadas quatro classes de uso e cobertura da terra, entre elas o Eucalipto cuja área aumentou de 4,4\% para 8,6\%. A classificação automática apresentou kappa de 0,79 e 0,8 , erros de quantidade de $2 \%$ e $3,5 \%$ e alocação de $5,5 \%$ e $5 \%$ para 200 I e $20 \mathrm{I}$ I, respectivamente. Conclui-se que o método de mineração de dados e as métricas de ecologia da paisagem foram eficientes na separação de classes de vegetação.

\section{GEOTECNOLOGY FOR FOREST COVER TEMPORAL ANALISYS}

ABSTRACT: The landscape ecology metrics associated with data mining can be used to increase the potential of remote sensing data analysis and applications, being an important tool for decision making. The present study aimed to use data mining techniques and landscape ecology metrics to classify and quantify different types of vegetation using a multitemporal analysis (200I and 20I I), in São Luís do Paraitinga city, São Paulo, Brazil. Object-based image analyses and the C4.5 data-mining algorithm were used for automated classification. Classification accuracies were assessed using the kappa index of agreement and the recently proposed allocation and quantity disagreement measures. Four land use and land cover classes were mapped, including Eucalyptus plantations, whose area increased from $4.4 \%$ to $8.6 \%$. The automatic classification showed a kappa index of 0.79 and 0.80 , quantity disagreements of $2 \%$ e $3.5 \%$ and allocation measures of $5.5 \%$ and $5 \%$ for $200 \mathrm{I}$ and $20 \mathrm{II}$, respectively. We therefore concluded that the data mining method and landscape ecology metrics were efficient in separating vegetation classes. 


\section{INTRODUÇÃO}

A vegetação representa um importante indicador das condições ambientais e contribui para manter a biodiversidade de uma região por proteger o solo, reduzir o transporte de sedimentos e o assoreamento dos cursos de água, além de servir de hábitat para animais silvestres. Entretanto, nas últimas décadas o processo de transformação da paisagem natural em uma paisagem antropizada intensificou a pressão sobre as áreas naturais, o que resulta em ambientes pouco diversificados, com fragmentos florestais isolados e de dimensões reduzidas (CALEGARI et al., 20l0).

No Brasil, o bioma da Mata Atlântica, com apenas II,76\% de sua cobertura original, possui uma longa história no uso da terra importante na compreensão dos processos resultantes da transformação dessas áreas (RIBEIRO et al., 2009; FREITAS et al., 2010) que vão desde eventos como enchentes, inundações até deslizamentos de terra.

Diante da problemática causada pelas alterações da cobertura vegetal, torna-se importante conhecer esta cobertura, bem como a estrutura da paisagem para auxiliar no monitoramento e planejamento do ambiente. Para isso, utilizam-se dados de sensoriamento remoto, como por exemplo, as imagens orbitais, para detectar, mapear e quantificar os alvos da superfície terrestre.

Entretanto, segundo Moreira (20II), deve-se tomar certos cuidados ao serem utilizadas imagens de média a baixa resolução espacial, como as imagens do sensor TM e do sensor Modis, para interpretar alvos na superfície terrestre. Como nestas imagens, para a maioria das vezes, não é possível individualizar o alvo, por exemplo, uma árvore, a interpretação geralmente é de forma dedutiva, ou seja, de forma indireta, em que o alvo é identificado através da associação das feições espectrais (comportamento espectral) e de outros elementos auxiliares, como forma, tamanho, textura, etc.

Diante disso, com o intuito de auxiliar na compreensão da complexidade de uma paisagem e a forma como esta influencia determinadas relações ecológicas, a estrutura da paisagem, obtida por meio de métricas de ecologia da paisagem, tem sido comumente utilizada como dado complementar aos estudos de interpretação de imagens (CARRÃO et al., 200I).

As métricas associadas às ferramentas computacionais, para extrair informações de imagens, como a mineração de dados espaciais e espectrais podem ser utilizadas para aumentar o potencial da análise e aplicações de dados de sensoriamento remoto
(KORTING et al., 2008) tornando-se ferramentas importantes para tomadas de decisões.

No presente estudo procurou utilizar técnicas de mineração de dados e métricas de ecologia da paisagem para classificação e quantificação de diferentes tipos de cobertura florestal presentes em uma análise multitemporal de imagens durante o período de 2001 a 20 I I, no município de São Luís do Paraitinga, São Paulo.

\section{MATERIAL E MÉTODOS}

\section{Área de estudo}

Esta pesquisa foi realizada no município de São Luís do Paraitinga (Figura I) localizado no estado de São Paulo, no Médio Vale do Paraíba do Sul, a uma latitude de

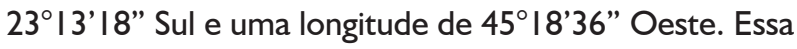
região, conhecida como Cone Leste Paulista, representa o berço da Bacia Hidrográfica do Rio Paraíba do Sul. Esta envolve algumas regiões de maior desenvolvimento e demografia do Brasil constituindo-se uma das bacias de maior relevância do país.

O município ocupa uma área de aproximadamente $62 \mathrm{I}, 4 \mathrm{Km}^{2}$, apresenta uma altitude média de 74I metros caracterizando-se geomorfologicamente por ser uma depressão de origem tectônica com declividade variável. Esta região é considerada de transição entre a floresta ombrófila densa (da encosta) e estacional semidecidual (do interior), com alguma influência da floresta ombrófila mista (CEIVAP, 20I I; IBGE, 20I I).

\section{METODOLOGIA}

Para realizar essa pesquisa foram utilizadas imagens TM/Landsat-5 (resolução espacial de 30 metros) referentes à órbita/ponto $218 / 76$, de 9 de setembro de $200 \mathrm{I}$ e de 5 de setembro de 201 I. O registro dessas cenas foi realizado com a imagem de referência disponível na base de dados Global Land Cover Facility: Earth Science Data Interface - GLFC (<http://glcfapp.glcf.umd. edu:8080/esdi/index.jsp >) de mesma órbita/ponto. Essa etapa foi realizada no aplicativo computacional ENVI 4.7, com ajuste polinomial de $1^{\circ}$ grau e erro menor que 0,5 pixel, ou seja, menor de $15 \mathrm{~m}$, o que é aceitável para esse tipo de análise. Todo o processo foi realizado em sistema de projeção UTM, zona 23 Sul e Datum WGS-84.

Imagens GeoEye de alta resolução espacial visualizadas por meio do aplicativo Google Earth 6. I.0.4857, foram utilizadas como mapa base para auxílio na interpretação dos alvos nas imagens TM/Landsat-5. 

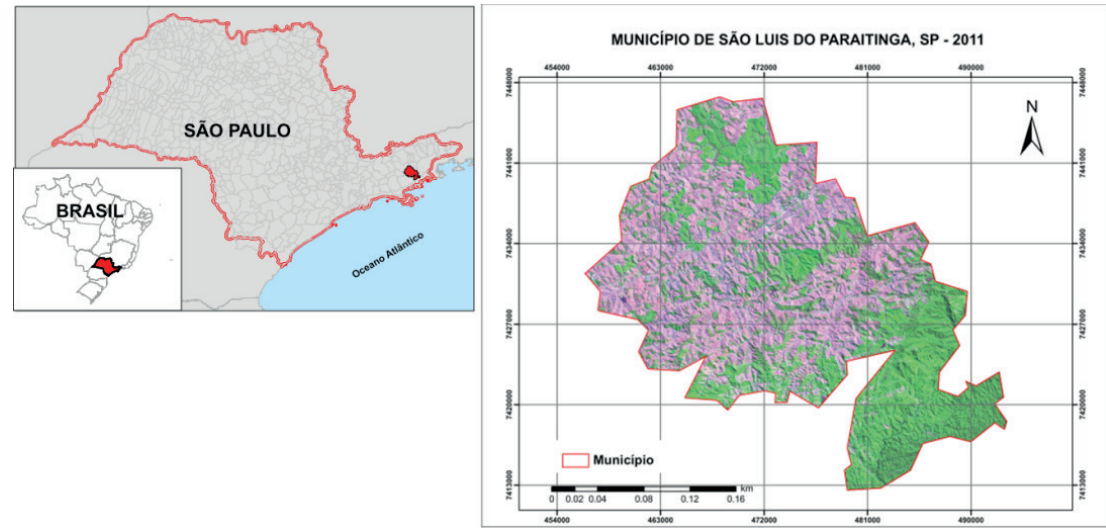

FIGURA 1 Representação da área de estudo por meio de composição colorida das bandas 5 (R) 4 (G) 3 (B) da imagem TM/ Landsat-5 de 2011.

FIGURE 1 Representation of the study area through color composite of bands 5 (R) 4 (G) 3 (B) of TM / Landsat-5 image of 2011.

Inicialmente, as imagens originais de 30 metros passaram por processo de correção atmosférica, por meio da correção radiométrica, utilizando a conversão das imagens em Número Digital para Reflectância no topo da atmosfera e posteriormente para reflectância de superfície, em que utilizou a técnica do pixel escuro (CHAVEZ, 1988). Ambas as operações foram realizadas no aplicativo computacional ENVI 4.7.

Nessa etapa também foi gerada a imagem NDVI (Normalized Difference Vegetation Index), com a finalidade de obter a máscara da cobertura florestal no município em estudo. Os valores do NDVI foram obtidos a partir da Equação I (Rouse et al., 1974)

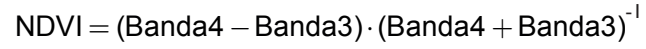

Após gerar as imagens NDVI com valores entre - I e I, aplicou-se um fatiamento do histograma para realçar apenas as áreas com vegetação. Para obter as áreas de cobertura florestal agrupou, por meio do fatiamento, os pixels da imagem cujos valores do NDVI situavam entre 0,55 a I,0.

Finalizando essa etapa, a máscara com a cobertura florestal do município recebeu um filtro de mediana 5 x 5 para retirar pixels isolados através da ferramenta (convolution) e o resultado foi transformado em vetores no formato de polígonos. Todo o processamento do NDVI foi realizado na imagem original $(30 \mathrm{~m})$ dentro do aplicativo ENVI 4.7.

Para facilitar a interpretação das classes de cobertura do solo nas imagens TM/Landsat, fez-se a restauração das mesmas (FONSECA, 1988) e a reamostragem para pixels de $10 \mathrm{~m}$, conforme Moreira (20II).

O processo de restauração, uma das técnicas de correção radiométrica, tem $\circ$ papel de estimar uma imagem que sofreu um processo de degradação
(MOREIRA, 20II). Dentre as diversas aplicações em sensoriamento remoto, a aproximação da imagem original sobre uma grade mais fina torna-se muitas vezes necessária, como no presente estudo, a fim de diferenciar as classes de cobertura vegetal. Porém, os resultados dos métodos de restauração, na prática, não são perfeitos, uma vez que informações (detalhes) são perdidas por algum tipo de espalhamento por luz e contaminação por ruído (FONSECA, 1988).

O procedimento foi realizado no aplicativo SPRING 4.3.3. (CÂMARA et al., 1996). Sobre as imagens restauradas aplicaram-se as máscaras de vegetação como demonstrado na Figura 2.

A segunda parte do trabalho foi realizada dentro do aplicativo computacional GeoDMA (Geographical Data Mining Analyst) (KORTING et al., 2008) para realizar uma análise orientada a objeto. Este sistema manipula e visualiza dados armazenados em bancos de dados geográficos (KORTING et al., 2008) e permite extrair dados espaciais e espectrais que são calculados e armazenados no aplicativo computacional TerraView.

Para $\circ$ presente estudo foram utilizadas 12 (doze) métricas entre espaciais e espectrais no GeoDMA, são elas: número de polígonos (NP), área (AREA), perímetro (PERIM), relação perímetroárea (PARA), circularidade (CIRCLE), índice de forma (SHAPE), retangularidade (RECT), elipticidade (ELLIPTIC), densidade, moda (MODE), media (MEAN) e desvio padrão (STDDEV).

Foram definidas quatro classes de cobertura vegetal, sendo elas: Eucalipto, Fragmento Florestal, Mata Ciliar e Outros (Figura 3). A classe Outros foi definida para aqueles alvos de difícil discriminação e com alta confusão na interpretação. 


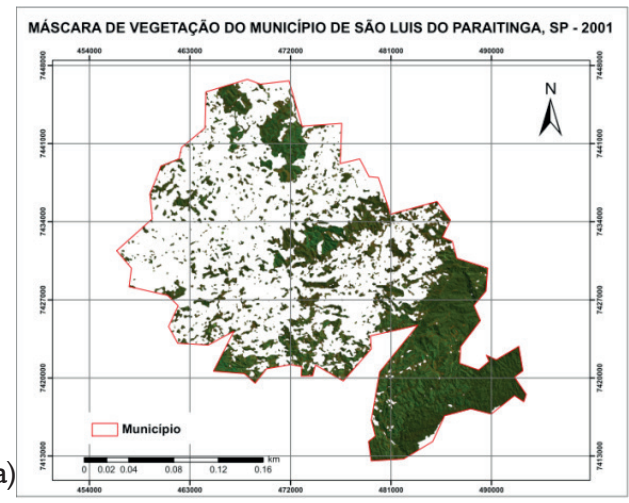

a)

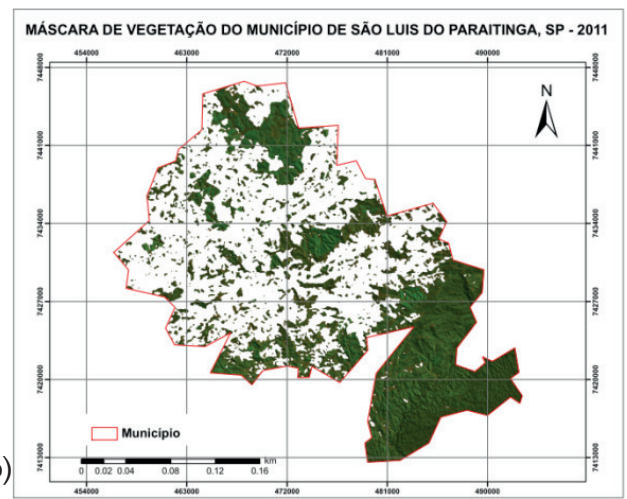

FIGURA 2 Máscaras de vegetação florestal nos anos de a) 2001 e b) 2011

FIGURE 2 Masks of forest vegetation in the years a) 2001 b) 2011

Aplicou-se o algoritmo de segmentação baseado no processo de crescimento de regiões, o qual utiliza, segundo Bins et al. (1996), a distância euclidiana e área mínima como parâmetros para dividir a imagem em regiões espectralmete homogêneas. Para isso foram testadas diversas segmentações, entretanto o limiar que melhor se enquadrou nos dados em análise foram os valores de 9 e 300 (pixels) para distância euclidiana e área mínima, respectivamente. Cada objeto gerado possui como atributo os valores calculados a partir das métricas selecionadas. $O$ próximo passo foi selecionar amostras de treinamento (Figura 3), que resultou num conjunto de informações. Estas amostras foram utilizadas para a classificação baseada em árvores de decisão pelo algoritmo C4.5.
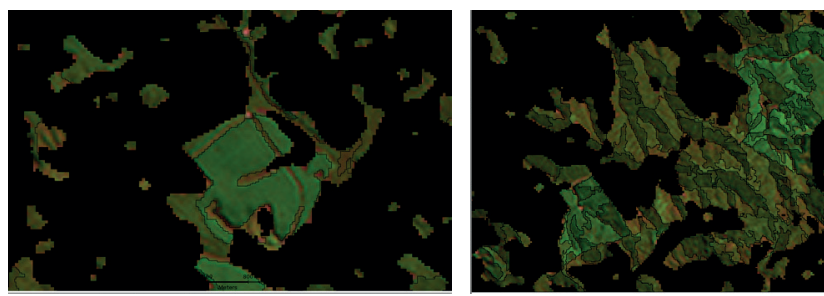

a. Eucalipto

b. Fragmento Florestal

FIGURA 3 Exemplos de amostras de treinamento.

FIGURE 3 Examples of training samples.

Para validar a classificação foram coletadas amostras de referência com auxílio das imagens GeoEye de alta resolução espacial. Essas amostras foram coletadas de forma estratificada regular (Figura 4) onde cada vértice de uma malha de $2 \times 2 \mathrm{~km}$ representou um ponto amostral, totalizaram 92 e 94 amostras para o ano de $200 \mathrm{l}$ e $20 \mathrm{ll}$.

Com as amostras de referência foi realizado, por meio da matriz de confusão, o cálculo do coeficiente de concordância Kappa (Equação 2), do Desempenho Geral (DG) (Equação 4), da Acurácia do Produtor (Equação 5) e da Acurácia do Usuário (Equação 6) para os anos de $200 \mathrm{l}$ e 20l I. Em que, $D$ é o elemento da diagonal principal e $T$ é o número total de amostras, representa $\circ$ total da linha para uma dada categoria informacional e representa o total da coluna para uma dada categoria informacional, $x_{i i}$ representa o elemento de determinada classe na linha i e coluna j.

$K=(D-Q) \cdot(D-T)^{-1}$

$Q=\sum_{j=1}^{r} \mathbf{X}_{i+} \mathbf{X}_{+i}$

$\mathrm{DG}=\mathrm{D} \cdot \mathrm{T}$

Acurácia do usuário $=\frac{x_{i j}}{x_{i+1}^{-1}}$

Acurácia do produtor $=\frac{x_{i j}}{x_{+j}^{-1}}$

Além disso, também foi avaliada a precisão da classificação por meio das medidas de discordância de alocação e de quantidade, recentemente propostas na literatura (PONTIUS JR; MILLONES, 20I I).

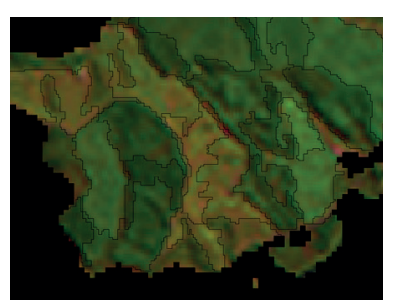

c. Outros

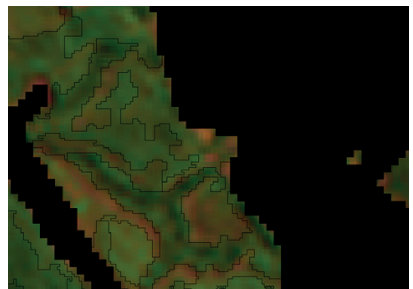

d. Mata Ciliar 


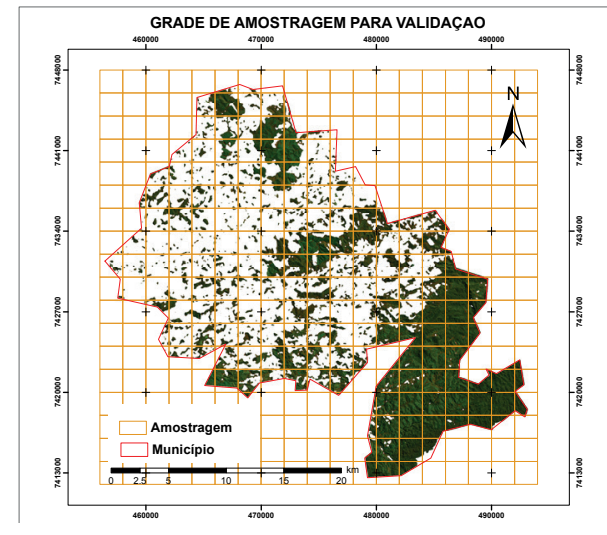

FIGURA 4 Grade de amostragem estratificada regular (alinhada) de $2 \times 2 \mathrm{~km}$.

FIGURE 4 Regular stratified sampling grid (aligned) of $2 \times 2 \mathrm{~km}$.

\section{RESULTADOS E DISCUSSÕES}

Após a realização das etapas iniciais deste trabalho, o classificador $\mathrm{C} 4.5$ gerou a partir das métricas calculadas as árvores de decisão utilizando as métricas que melhor se enquadraram para cada conjunto de dados (Figura 5).

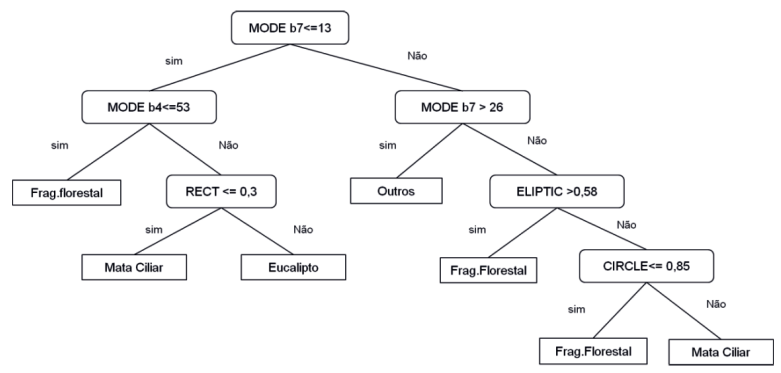

a.

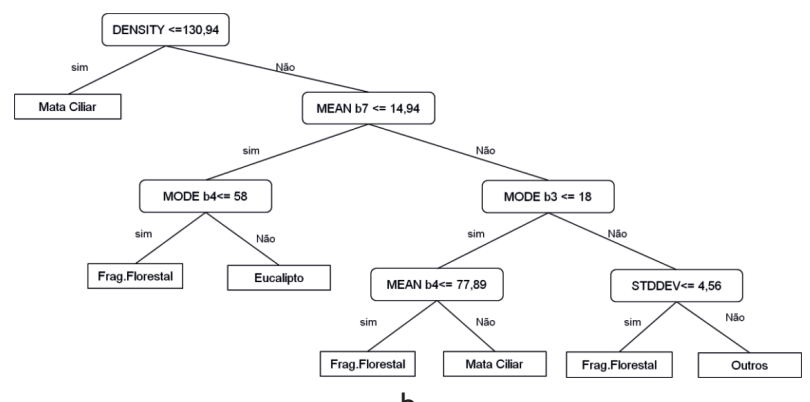

b.

FIGURA 5 Árvores de decisão para o ano de 2001(a) e 2011(b). FIGURE 5 Decision Trees for the year 2001(a) and 2011 (b).

Utilizando alguns dos atributos fornecidos pelas árvores de decisão foram gerados os gráficos de dispersão das classes (Figura 6) para melhor compreender como ocorre a separação entre elas. Observou-se em $200 \mathrm{I}$ que as classes Eucalipto, Fragmento Florestal e Outros apresentaram valores similares de RECT, porém encontraram-se distribuídas em camadas no eixo y (MODE), o que favorece a individualização das mesmas. Considerando que os valores de RECT próximos de I indicam que o objeto é mais retangular não foi possível a separação das classes somente com esse atributo, uma vez que o ano de 200 I apresentou classes regularmente distribuídas no parâmetro RECT.

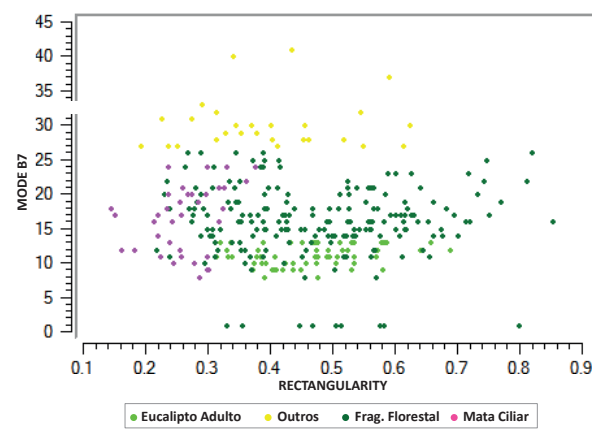

a. MODE b7 x RECTANGULARITY - 200I

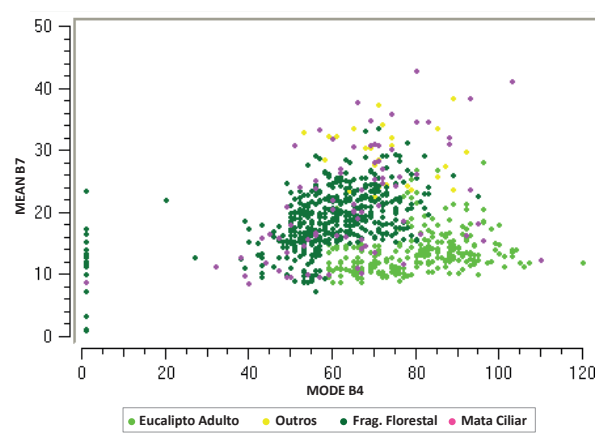

b. MEAN B7 X MODE B4 - 201 I

FIGURA 6 Gráfico de dispersão de atributos espectrais e espaciais: a) 2001 e b) 2011.

FIGURE 6 Scatter plot using spectral and spatial attributes: a) 2001 b) 2011.

Já para o ano de $201 \mathrm{I}$ utilizaram-se as métricas espectrais média da banda 7 e moda da banda 4, sendo possível visualizar apenas as classes Eucalipto e Fragmento Florestal, confirmando a necessidade de outros parâmetros para individualizar todas as classes.

Utilizando as árvores de decisão geradas pelo algoritmo de classificação obtiveram-se os mapas de cobertura florestal, apresentados na Figura 7, com base na máscara de vegetação inserida nas etapas anteriores dessa pesquisa. Com as Figuras 7 e 8 e a Tabela I observa-se que nos anos analisados a principal alteração encontrada sobre a cobertura florestal refere-se ao aumento de 4,4\% para 8,6\% das áreas com Eucalipto, uma diminuição de $36,3 \%$ para $36,1 \%$ das áreas com 


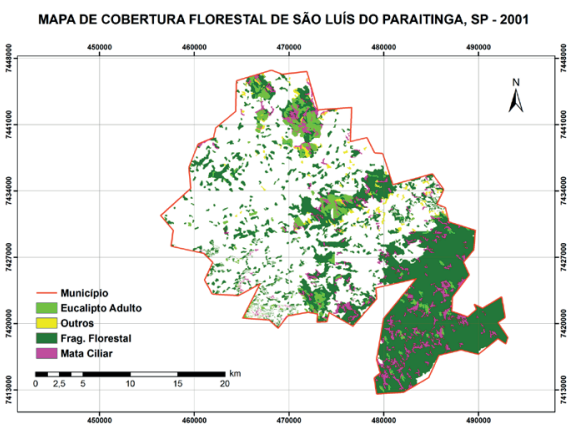

a. 2001

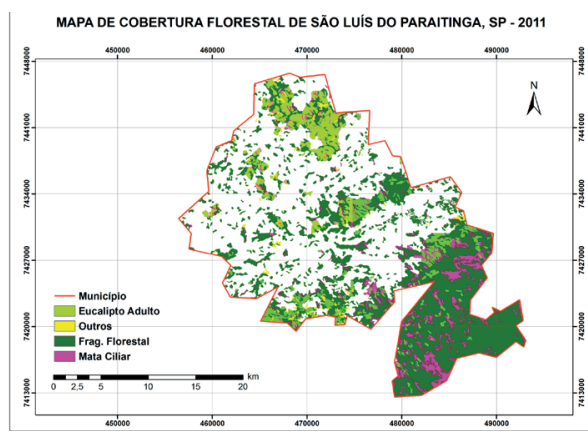

b. 2011

FIGURA 7 Mapas de cobertura florestal de São Luís do Paraitinga, SP.

FIGURE 7 Forest cover maps of Sao Luís do Paraitinga, SP.

Fragmentos Florestais e o crescimento da Mata Ciliar de $4,7 \%$ para $5,3 \%$.

Por meio de análises espaciais para detecção de mudança, observaram-se os ganhos e perdas das classes mapeadas no município (Figura 9). Ao longo do período estudado nota-se incremento da classe Eucalipto efetuado, principalmente, sobre áreas de floresta e áreas sem vegetação (fora da máscara de vegetação). Sobre a classe Floresta os ganhos e perdas foram equivalentes, com 2612,53 ha de áreas de Eucalipto cederam espaço para a formação de Fragmentos Florestais e 2271,94

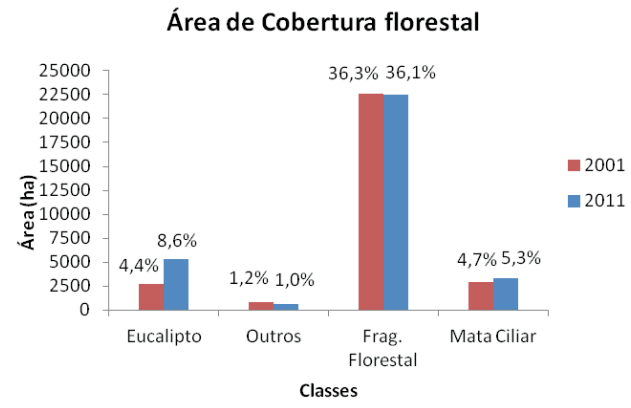

FIGURA 8 Área (ha e \%) da cobertura florestal no município de São Luís do Paraitinga, SP

FIGURE 8 Area (ha and \%) of forest cover in São Luís do Paraitinga, SP. ha de Floresta tornaram-se cultivos econômicos de Eucalipto ao longo dos II anos analisados.

Os ganhos de áreas de Eucalipto sobre áreas sem vegetação podem ser observados pela Figura 9d, na qual indica a perda de área da classe Sem vegetação para a classe Eucalipto, ou seja, áreas com pouca ou nenhuma cobertura vegetal foram transformadas em cultivos econômicos. Apesar do aumento de cultivos econômicos, a cobertura do solo pela vegetação arbórea é preferível a um pasto mal manejado ou um solo exposto para a conservação dos recursos hídricos.

Um ponto positivo dessa análise, apesar de não ter ocorrido aumento na área total dos fragmentos florestais observa-se que a classe Floresta teve um ganho expressivo de área sobre a classe Sem vegetação.

Além disso, pela análise das demais métricas da paisagem calculadas, verificou-se que não somente a área de Eucalipto aumentou como também o número

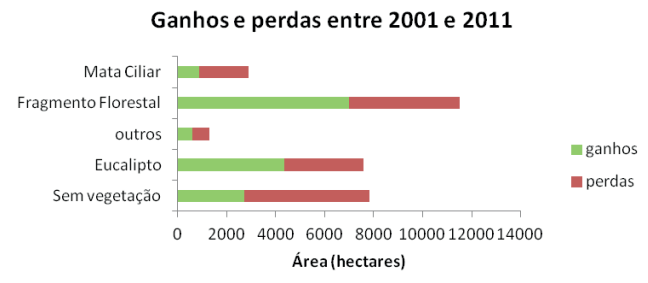

a)

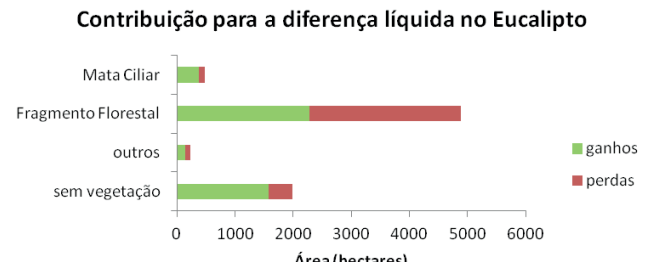

b)

Contribuição para a diferença líquida no Floresta

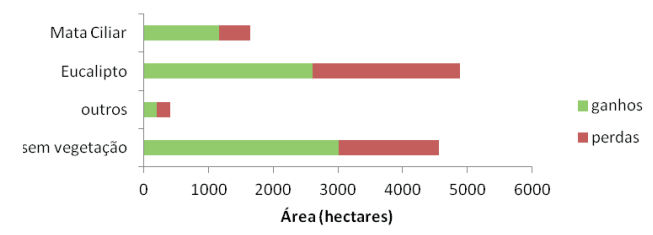

Contribuição para a diferença líquida Sem Vegetação

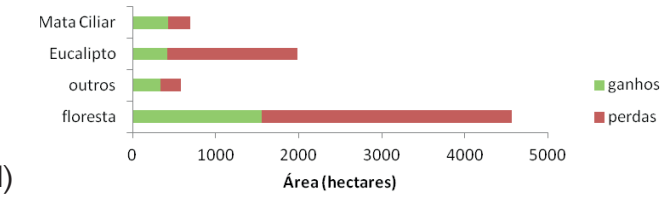

FIGURA 9 Detecção de mudanças do uso e cobertura da terra no município de São Luís do Paraitinga, SP.

FIGURE 9 Land use and land cover change detection in São Luís do Paraitinga, SP. 
TABELA 1 Médias das métricas de paisagem.

TABLE 1 Mean values of landscape metrics.

\begin{tabular}{ccccccc}
\hline Classes Imétricas & NP & AREA $\left(\mathrm{m}^{2}\right)$ & CIRCLE & PERIM $(\mathrm{m})$ & PARA & SHAPE \\
\hline Eucalipto Adulto & 269,00 & 95202,30 & 0,76 & 2581,44 & 0,15 & 2,15 \\
Outros & 108,00 & 36501,55 & 0,38 & 1126,64 & 0,02 & 1,05 \\
Frag. Floresta & 1972,00 & 106597,74 & 0,73 & 2649,46 & 0,14 & 2,06 \\
Mata Ciliar & 343,00 & 81062,20 & 0,89 & 3248,96 & 0,09 & 2,87 \\
\hline Eucalipto Adulto & 486,00 & 104522,29 & 0,75 & 2638,75 & 0,03 & 2,11 \\
Outros & 80,00 & 60888,14 & 0,58 & 1928,72 & 0,03 & 1,70 \\
Frag. Floresta & 1971,00 & 106879,90 & 0,74 & 2699,04 & 0,03 & 2,09 \\
Mata Ciliar & 354,00 & 67152,60 & 0,91 & 2716,48 & 0,05 & 2,76 \\
\hline
\end{tabular}

de polígonos dessa classe, indicando a expansão de áreas cultivadas com essa essência florestal no município durante o período de análise. Esses dados confirmam o trabalho de Arguello (2010) que indicaram o incremento de eucalipto nesse município nos períodos entre 2000 a 2007 e 2007 a 2010 , respectivamente.

Análises dessa qualidade são importantes para a gestão pública, bem como no monitoramento, aplicação e fiscalização da ação pública implementada pelo instrumento $n^{\circ} 759.170-5 / 3-00$ TJSP, de 28 de março de 2008, cujo objetivo era conter os impactos e os problemas ocasionados pela expansão dos cultivos de Eucalipto no município.

As métricas CIRCLE, PARA E SHAPE são importantes para compreender a estrutura dos polígonos e como esses se relacionam com o ambiente. Em geral, em ambos os anos analisados, as classes apresentaram forma mais irregulares com valores de SHAPE afastando-se de I (valores iguais a I indicam forma mais compacta). Contudo, estas se apresentaram com pouca complexidade de forma com valores de PARA próximo de zero. Essas métricas são confirmadas também pela CIRCLE, cujos valores próximos a I afastam a forma de um círculo (Tabela I).

Vale ressaltar que a grande porcentagem de fragmentos florestais da Mata Atlântica encontrada nesse município confirmou o exposto por Ribeiro et al. (2009), cujas áreas desse bioma que ainda estão preservados são decorrentes da dificuldade de acesso como no caso da Serra do Mar, localizada a Sudeste da área de estudo.

A validação da classificação realizada pelo algoritmo C4.5 por meio do índice Kappa resultou em valores de 0.79 para o ano de $200 \mathrm{I}$ e 0.80 para $20 \mathrm{II}$, a partir da matriz de confusão que pode ser observada pelas Tabelas 2. Apesar de classificações sobre esse índice estarem reduzidas, segundo Cogalton e Green (1998), esses valores indicaram uma classificação razoável.

Os resultados obtidos com o índice kappa são complementados com a Exatidão Global de 9/\% e $90 \%$ para $200 \mathrm{I}$ e $20 \mathrm{II}$, respectivamente e com os altos valores das acurácias do produtor e usuário (Tabela 3).

Baixos valores das medidas de discordância de alocação e de quantidade obtidos para ambas as datas de 200 I e 20 I I , 5,5\% e 5\%; $2 \%$ e $3,5 \%$, respectivamente, corroboraram os resultados dos índices anteriores. Entretanto, Pontius Jr. e Millones (20I I), recomendam que o índice Kappa seja abandonado pelos profissionais da área de sensoriamento remoto, uma vez que esse índice apresenta limitações, falhas e resultados enganadores. Em contrapartida apresentam e demonstram que as medidas de discordância de alocação e de quantidade são facilmente obtidas pela matriz de confusão e os resultados são mais realísticos e úteis.

TABELA 2 Matriz de confusão para 2001 e 2011

TABLE 2 Confusion matrix for 2001

\begin{tabular}{|c|c|c|c|c|c|c|c|c|c|c|}
\hline \multirow[b]{2}{*}{ Classes } & \multicolumn{5}{|c|}{ Classificação 2001} & \multicolumn{5}{|c|}{ Classificação 2011} \\
\hline & Eucalipto & Outros & Frag. Florestal & Mata Ciliar & Total & Eucalipto & Outros & Frag. Florestal & Mata Ciliar & Total \\
\hline Eucalipto & 10 & 0 & 0 & 0 & 10 & 17 & 0 & 1 & 1 & 19 \\
\hline Outros & 0 & 3 & 1 & 0 & 4 & 2 & 3 & 0 & 0 & 5 \\
\hline Frag. Florestal & 1 & 1 & 65 & 2 & 69 & 1 & 0 & 60 & 3 & 64 \\
\hline Mata Ciliar & 1 & 0 & 2 & 6 & 9 & 0 & 0 & 1 & 5 & 6 \\
\hline Total & 12 & 4 & 68 & 8 & 92 & 20 & 3 & 62 & 9 & 94 \\
\hline
\end{tabular}


TABELA 3 Acurácia do produtor e do usuário para 2001 e 2011

TABLE 3 Producer and user accuracy for 2001 and 2011

\begin{tabular}{ccccccccc}
\hline & \multicolumn{2}{c}{ Eucalipto (\%) } & \multicolumn{2}{c}{ Outros $(\%)$} & \multicolumn{2}{c}{ Frag. Florestal (\%) } & \multicolumn{2}{c}{ Mata Ciliar (\%) } \\
\hline & 2001 & 2011 & 2001 & 2011 & 2001 & 2011 & 2001 & 2011 \\
\hline $\begin{array}{c}\text { Acurácia Produtor } \\
\left(\mathrm{x}_{\mathrm{i}} / \mathrm{x}_{\mathrm{i}+}\right)\end{array}$ & 83,3 & 85,0 & 75 & 100 & 95,5 & 96,7 & 75,0 & 55,5 \\
$\begin{array}{c}\text { Acurácia Usuário } \\
\left(\mathrm{x}_{\mathrm{i}} / \mathrm{x}_{+\mathrm{i}}\right)\end{array}$ & 100,0 & 89,4 & 75 & 60 & 94,2 & 93,7 & 66,6 & 83,3 \\
\hline
\end{tabular}

\section{CONCLUSÕES}

O aplicativo computacional GeoDMA, em suas atribuições, ao extrair atributos espaciais e espectrais por meio de mineração de dados e ecologia da paisagem como dados de entrada para o algoritmo de classificação C4.5, apresentou-se como uma ferramenta acessível e confiável para classificação de imagens provenientes de sensores remotos.

Através desta pesquisa foi possível verificar a dinâmica das mudanças de uso e cobertura da terra e, principalmente, a expansão das áreas de eucalipto e as classes envolvidas no processo.

As métricas utilizadas permitiram compreender melhor os objetos inseridos na paisagem e as pressões que estes sofrem, tal como as alterações ocorridas na cobertura florestal do município de São Luís do Paraitinga, SP entre o período analisado de 200 I e 201 I.

\section{REFERÊNCIAS}

ARGUELLO, F. V. P. Expansão do eucalipto no trecho paulista da bacia hidrográfica Paraíba do Sul. 2010. 79 p. Dissertação (Mestrado em Ciências Ambientais) Universidade de Taubaté, Programa de Pós-graduação em Ciências Ambientais, Taubaté. 2010.

BINS, L.; FONSECA, L. M.; ERTHAL, G. J.; MITSUO II, F. "Satellite Imagery Segmentation: a Region Growing Approach". In: Simpósio Brasileiro de Sensoriamento Remoto, 8., 14-19 abr. 1996, Salvador, Brasil, Anais... São José dos Campos: INPE, p. 677-680.

CALEGARI, L.; MARTINS, S. V.; GLERIANI, J. M.; SILVA, E.; BUSATO, L. C. Análise da dinâmica de fragmentos florestais no município de Carandaí, MG, para fins de restauração florestal. Revista Árvore, Viçosa-MG, v.34, n.5, p.87I$880,2010$.

CÂMARA, G.; SOUZA, R. C. M.; FREITAS, U. M.; GARRIDO, J. SPRING: Integrating remote sensing and GIS by objectoriented data modeling. Computers \& Graphics, v.20, n.3, p.395-403, 1996.
CEIVAP. Comitê de Integração da Bacia Hidrográfica do Rio Paraíba do Sul. Dados geoambientais. Disponível em: < http:// ceivap.org.br/bacia_I_2.php>. Acesso em: 08 out. 201 I.

CONGALTON, R. G.; GREEN, K. Assessing the accuracy of remotely sensed data: principles and practices. New York: Lewis Publishers, 1998. 137p.

FREITAS, S. R.; HAWBAKER, T. J.; METZGER, J. P. Effects of roads, topography, and land use on forest cover dynamics in the Brazilian Atlantic Forest. Forest Ecology and Management. v. 259, p. 4I0-4I7, 2010.

FONSECA, L.M.G. Restauração e interpolação de imagens do satélite Landsat por meio de técnicas de projeto de filtros FIR. São José dos Campos. 148 p.(INPE-6628$\mathrm{TAE} / 30$ ). Dissertação (Mestrado em Engenharia Elétrica) Instituto Tecnológico da Aeronáutica, 1988.

IBGE. Instituto Brasileiro de Geografia e Estatística. Banco de Dados Agregados. Disponível em: < http://www.ibge. gov.br>. Acesso: em: 08 out. 2011 .

KORTING, T. S.; FONSECA, L. M.; ESCADA, M. I. S.; SILVA, F. C.; SILVA, M. P. S. GeoDMA: a novel system for spatial data mining. IEEE International Conference on Data Mining Workshops, 2008.

MOREIRA, M. A. Fundamentos do sensoriamento remoto e metodologias de aplicação. 4 ed, Viçosa: UFV, 20I I, 422p.

PONTIUS, R. G. JR.; MILLONES, M. Death to Kappa: birth of quantity disagreement and allocation disagreement for accuracy assessment. International Journal of Remote Sensing, v.32, p.4407-4429, $201 \mathrm{l}$.

RIBEIRO, M. C.; METZGER, J. P.; MARTENSEN, A. C.; PONZONI, F. J.; HIROTA, M.M. The Brazilian Atlantic Forest: How much is left, and how is the remaining forest distributed? Implications for conservation. Biological Conservation, v.I42, p.II4I-II53, 2009.

ROUSE, J. W.; HAAS, R. H.; SCHELL, J. A.; DEERING, D. W.; HARLAN, J. C. Monitoring the vernaladvancement of retrogradation (greenwave effect) of natural vegetation. NASA/GSFC, Type III, FinalReport, Greenbelt, MD, 1974, 37I p. 